\title{
The Bistability Theorem in a Model of Metastatic Cancer
}

\author{
Jens Christian Larsen \\ Vanløse Alle 502 mf tv, 2720 Vanløse, Copenhagen, Denmark \\ Email: jlarsen.math@hotmail.com \\ Received 2 May 2016; accepted 27 June 2016; published 30 June 2016 \\ Copyright (C) 2016 by author and Scientific Research Publishing Inc. \\ This work is licensed under the Creative Commons Attribution International License (CC BY). \\ http://creativecommons.org/licenses/by/4.0/ \\ (c) (i) Open Access
}

\section{Abstract}

The main theorem of the present paper is the bistability theorem for a four dimensional cancer model, in the variables $C, C_{M}, G F, G I$ representing primary cancer $C$, metastatic cancer $C_{M}$, growth factor $G F$ and growth inhibitor $G I$, respectively. It says that for some values of the parameters this system is bistable, in the sense that there are exactly two positive singular points of this vector field. And one is stable and the other unstable. We also find an expression for $\frac{\mathrm{d} C}{\mathrm{~d} t}(0)$ for the discrete model $T$ of the introduction, with variables $(C, G F, G I)$, where $C$ is cancer, $G F, G I$ are growth factors and growth inhibitors respectively. We find an affine vector field $Y$ whose time one map is $T^{2}$ and then compute $\frac{d C}{d t}(0)$, where $(C(t), G F(t), G I(t))$ is an integral curve of $Y$ through $\left(0, G F_{0}, G I_{0}\right) \in \mathbb{R}^{3}$. We also find a formula for the first escape time for the vector field associated to $T$, see section four.

Keywords

Bistability, Cancer, Mass Action Kinetic System, Discrete Dynamical System

\section{Introduction}

\subsection{Summary of the Paper}

We continue the study of the cancer model from Larsen (2016) [1]. The model is

$$
T: \mathbb{R}^{3} \rightarrow \mathbb{R}^{3}
$$




$$
T(y)=A y+g+c
$$

where

$$
A=\left(\begin{array}{ccc}
1+\gamma & \alpha & \beta \\
\delta & 1+\mu_{F} & 0 \\
\sigma & 0 & 1+\mu_{I}
\end{array}\right)
$$

$g=\left(g_{C}, g_{F}, g_{I}\right)^{\mathrm{T}} \in{\overline{\mathbb{R}_{+}}}^{3}$ are birth rates and $T$ denotes transpose. Here $c=\left(c_{1}, 0,0\right)^{\mathrm{T}}, c_{1}<0$ is chemotherapy and $c=\left(0,0, c_{3}\right)^{\mathrm{T}}, c_{3}>0$ is immune therapy. The parameters $\gamma, \delta, \sigma \in \mathbb{R}, \alpha \in \mathbb{R}_{+}, \mu_{F}, \mu_{I} \in \overline{\mathbb{R}_{-}}, \beta \in \mathbb{R}_{-}$. We have shown previously Larsen (2016) [1], that there are affine vector fields on $\mathbb{R}^{3}$, such that their time one map is $T$, when the eigenvalues of $A$ have positive real part. This enables you to find a formula for the rate of change of cancer growth in $C=0$. The characteristic polynomial of $A$ is

$$
(1-\lambda)((1-\lambda)(1+\gamma-\lambda)-\alpha \delta-\beta \sigma)
$$

when $\mu_{F}=\mu_{I}=0$. The discriminant of this polynomial is

$$
\nabla=\gamma^{2}+4(\alpha \delta+\beta \sigma)
$$

The eigenvalues are

$$
\lambda_{ \pm}=\frac{2+\gamma}{2} \pm \frac{1}{2} \sqrt{\nabla} \quad \lambda=1
$$

In section two we prove the Bistability Theorem for a mass action kinetic system of metastatic cancer $C_{M}$ and primary cancer $C$. The model also has GF growth factors and GI growth inhibitors. We show that for some values of the parameters there are exactly two positive singular points $C_{*}^{-}=\left(C_{*}^{-}, C_{*}^{M-}, G F_{*}, G I_{*}^{-}\right)$, $c_{*}^{+}=\left(C_{*}^{+}, C_{*}^{M+}, G F_{*}, G I_{*}^{+}\right)$, where $C_{*}^{-}<C_{*}^{+}, C_{*}^{M-}<C_{*}^{M+}$. We prove that $C_{*}^{+}$is unstable and $C_{*}^{-}$is stable, when one of the rate constants is small.

For $\nabla<0$ we have: if the eigenvalue $a+i b$ of $A$ has $a^{2}>b^{2}$ then one can find an affine vector field, whose time one map is $T^{2}$. Similarly, when $\nabla>0, \alpha \delta+\beta \sigma<0$ and the eigenvalues $\lambda_{-}, \lambda_{+}$of the characteristic polynomial of $A$ are nonzero, then one can find an affine vector field on $\mathbb{R}^{3}$, whose time one map is $T^{2}$. This enables us to find a formula for the rate of change of cancer growth in $C=0$. This is the subject of Section 3.

The phase space of our model $T$ is $\mathbb{R}_{+}^{3}$. In section four we show, that when $\mu_{F}=\mu_{I}=0, g=0, \nabla<0$, $\mathfrak{R}\left(\lambda_{+}\right)>0$ orbits of the vector field associated to $T$ will escape phase space for both $t>0$ and $t<0$. We obtain a formula for the first escape time. There is a similar treatment for $\nabla>0$.

\subsection{The Litterature}

uPAR (urokinase plasminogen activator receptor) is a cell surface protein, that is associated with invasion and metastasis of cancer cells. In Liu et al. (2014) [2] a cytoplasmic protein Sprouty1 (SPRY1) an inhibitor of the (Ras-mitogen activated protein kinase) MAPK pathway is shown to interact with uPAR and cause it to be degraded. Overexpression of SPRY1 in HCT116 or A549 xenograft in athymic nude mice, led to great suppression of tumor growth. SPRY1 is an inhibitor of the MAPK pathway. Several cancer cells have a low basal expression of SPRY1, e.g. breast, prostate and liver cancer. SPRY1 promotes the lysosomal mediated degradation of uPAR. SPRY1 overexpression results in a decreased expression of UPAR protein. This paper suggests that SPRY1 regulates cell adhesion through an uPAR dependant mechanism. SPRY1 inhibits proliferation via two distinct pathways: 1) SPRY1 is an intrinsic inhibitor of the Raf/MEK/ERK pathway; 2) SPRY1 promotes degradation of uPAR, which leads to inhibition of FAK and ERK activation.

According to Luo and Fu (2014), [3] EGFR (endoplasmic growth factor receptor) tyrosine kinase inhibitors (TKIs) are very efficient against tumors with EGFR activating mutations in the EGFR intracytoplasmic tyrosin kinase domain and cell apoptosis was the result. However some patients developed resistance and this reference aimed to elucidate molecular events involved in the resistance to EGFR-TKIs. The first EGFR-TKI s to be 
approved by the FDA (Food and Drug Administration, USA) for treatment of NSCLC (non small cell lung cancer) were gefitinib and erlotinib. The mode of action is known. These drugs bind to the ATP binding site of EGFR preventing autophosphorylation and then blocking downstream signalling cascades of pathways RAS/ RAF/MEK/ERK and PI3K/AKT with the results, proliferation inhibition, cell cycle progression delay and cell apoptosis.

There are several important monographs relevant to the present paper, see Adam \& Bellomo (1997), [4], Geha \& Notarangelo (2012), [5], Murphy (2012), [6], Marks (2009), [7], Molina (2011), [8].

\section{A mass Action Kinetic Model of Metastatic Cancer}

The main result of this section is Theorem 1 below that proves the bistability of the mass action kinetic system (1) to (8). Consider then the mass action kinetic system from Larsen (2016), [9], in the species $C, C_{M}, G F, G I$ primary cancer cells, metastatic cancer cells, growth factor, growth inhibitor respectively.

$$
\begin{aligned}
& G F \rightarrow C \\
& G F \rightarrow C_{M} \\
& C+G I \rightarrow 0 \\
& C_{M}+G I \rightarrow 0 \\
& C \rightarrow 2 C \\
& C_{M} \rightarrow 2 C_{M} \\
& 0 \leftrightarrows G F \\
& 0 \leftrightarrows G I
\end{aligned}
$$

The complexes are $C(1)=G F, C(2)=C, C(3)=C_{M}, C(4)=C+G I, C(5)=0, C(6)=C_{M}+G I$, $C(7)=2 C, C(8)=2 C_{M}, C(9)=G I$. And this defines the rate constants $k_{i j}$. With mass action kinetics the ODE s become

$$
\begin{gathered}
C^{\prime}=k_{21} G F-k_{54} C \cdot G I+k_{72} C \\
C_{M}^{\prime}=k_{31} G F-k_{56} C_{M} \cdot G I+k_{83} C_{M} \\
G F^{\prime}=-\left(k_{21}+k_{31}+k_{51}\right) G F+k_{15} \\
G I^{\prime}=-k_{54} C \cdot G I-k_{56} C_{M} \cdot G I+k_{95}-k_{59} G I
\end{gathered}
$$

all $k_{i j}>0$. We shall now find the singular points of this vector field denoted

$$
f: \mathbb{R}^{4} \rightarrow \mathbb{R}^{4}
$$

But first we state a theorem, we shall next prove. A positive (nonnegative) singular point $\left(C, C_{M}, G F, G I\right)$ of $f$ is a singular point of $f$, such that $\left(C, C_{M}, G F, G I\right) \in \mathbb{R}_{+}^{4}, \quad\left(\left(C, C_{M}, G F, G I\right) \in \overline{\mathbb{R}}_{+}^{4}\right)$. Define

$$
k_{1}=k_{21} \frac{k_{15}}{k_{21}+k_{31}+k_{51}}, \quad k_{2}=k_{31} \frac{k_{15}}{k_{21}+k_{31}+k_{51}}
$$

Theorem 1 Assume $k_{72}=k_{54}, k_{83}=k_{56}$. When $k=k_{95}-k_{59}-k_{1}-k_{2}>0, \quad \nabla=k^{2}-4 k_{59}\left(k_{1}+k_{2}\right)>0$, there are exactly two positive singular points

$$
c_{*}^{-}=\left(C_{*}^{-}, C_{*}^{M-}, G F_{*}, G I_{*}^{-}\right) \quad c_{*}^{+}=\left(C_{*}^{+}, C_{*}^{M+}, G F_{*}, G I_{*}^{+}\right)
$$

where $C_{*}^{-}<C_{*}^{+}, C_{*}^{M-}<C_{*}^{M+}$. $C_{*}^{+}$is unstable. Given $k_{95}=k_{95}^{0}, k_{1}=k_{1}^{0}, k_{2}=k_{2}^{0}, k_{59}=k_{59}^{0} \in \mathbb{R}_{+}$, such that $k=k_{0}=k_{95}^{0}-k_{59}^{0}-k_{1}^{0}-k_{2}^{0}>0$ and, $\nabla^{0}=k_{0}^{2}-4 k_{59}^{0}\left(k_{1}^{0}+k_{2}^{0}\right)>0$, then there exists $\epsilon>0, \epsilon<k_{59}^{0}$ such that $C_{*}^{-}$ 
is stable when $k_{59}<\epsilon$.

Consider a singular point $C_{*}=\left(C_{*}, C_{*}^{M}, G F_{*}, G I_{*}\right)$ of $f$ and linearize

$$
B=D f_{c_{*}}=\left(\begin{array}{cccc}
k_{72}-k_{54} G I_{*} & 0 & k_{21} & -k_{54} C_{*} \\
0 & k_{83}-k_{56} G I_{*} & k_{31} & -k_{56} C_{*}^{M} \\
0 & 0 & -\left(k_{21}+k_{31}+k_{51}\right) & 0 \\
-k_{54} G I_{*} & -k_{56} G I_{*} & 0 & -k_{59}-k_{54} C_{*}-k_{56} C_{*}^{M}
\end{array}\right)
$$

Setting the last coordinate of $f$ equal to zero gives

$$
G I_{*}=\frac{k_{95}}{k_{54} C_{*}+k_{56} C_{*}^{M}+k_{59}}
$$

when $C_{*}, C_{*}^{M} \geq 0$. Now insert this into the first and second coordinates of $f$ to get

$$
k_{1}\left(k_{54} C_{*}+k_{64} C_{*}^{M}+k_{59}\right)-k_{54} k_{95} C_{*}+k_{72} C_{*}\left(k_{54} C_{*}+k_{64} C_{*}^{M}+k_{59}\right)=0
$$

and

$$
k_{2}\left(k_{54} C_{*}+k_{64} C_{*}^{M}+k_{59}\right)-k_{56} k_{95} C_{*}^{M}+k_{83} C_{*}^{M}\left(k_{54} C_{*}+k_{64} C_{*}^{M}+k_{59}\right)=0
$$

When $C_{*}, C_{*}^{M}>0$ we get from (9)

$$
\frac{k_{1}+k_{72} C_{*}}{C_{*}}=k_{54} G I_{*}=\frac{k_{54} k_{95}}{k_{54} C_{*}+k_{56} C_{*}^{M}+k_{59}}
$$

and from (10) we get

$$
\frac{k_{2}+k_{83} C_{*}^{M}}{C_{*}^{M}}=k_{56} G I_{*}=\frac{k_{56} k_{95}}{k_{54} C_{*}+k_{56} C_{*}^{M}+k_{59}}
$$

This means that $B$ simplifies to

$$
B=D f_{c_{*}}=\left(\begin{array}{cccc}
-\frac{k_{1}}{C_{*}} & 0 & k_{21} & -k_{54} C_{*} \\
0 & -\frac{k_{2}}{C_{*}^{M}} & k_{31} & -k_{56} C_{*}^{M} \\
0 & 0 & -\left(k_{21}+k_{31}+k_{51}\right) & 0 \\
-k_{54} G I_{*} & -k_{56} G I_{*} & 0 & -k_{59}-k_{54} C_{*}-k_{56} C_{*}^{M}
\end{array}\right)
$$

Let $\tilde{B}$ denote the matrix you obtain by deleting row three and column three in $B$. Then

$$
\begin{aligned}
\operatorname{det} \tilde{B}= & -\frac{k_{1} k_{2}}{C_{*} C_{*}^{M}}\left(k_{59}+k_{54} C_{*}+k_{56} C_{*}^{M}\right) \\
& +\frac{k_{2} k_{54}}{C_{*} C_{*}^{M}} C_{*}\left(k_{1}+k_{72} C_{*}\right)+\frac{k_{1} k_{56}}{C_{*} C_{*}^{M}} C_{*}^{M}\left(k_{2}+k_{83} C_{*}\right) \\
= & \frac{-k_{1} k_{2} k_{59}+k_{2} k_{54} k_{72} C_{*}^{2}+k_{1} k_{56} k_{83} C_{*}^{M 2}}{C_{*} C_{*}^{M}}
\end{aligned}
$$

Also

$$
\sigma=\operatorname{trace} \tilde{B}=-\frac{k_{1}}{C_{*}}-\frac{k_{2}}{C_{*}^{M}}-\left(k_{59}+k_{54} C_{*}+k_{56} C_{*}^{M}\right)
$$

The characteristic polynomial of $\tilde{B}$ is denoted 


$$
\operatorname{det}(\tilde{B}-\lambda \mathrm{id})=-\lambda^{3}+\sigma \lambda^{2}-\tau \lambda+\delta
$$

Finally

$$
\begin{aligned}
\tau= & \frac{k_{1} k_{2}}{C_{*} C_{*}^{M}}+\frac{k_{1}}{C_{*}}\left(k_{59}+k_{54} C_{*}+k_{56} C_{*}^{M}\right)-\frac{k_{1}+k_{72} C_{*}}{C_{*}} k_{54} C_{*} \\
& +\frac{k_{2}}{C_{*}^{M}}\left(k_{59}+k_{54} C_{*}+k_{56} C_{*}^{M}\right)-\frac{k_{2}+k_{83} C_{*}^{M}}{C_{*}^{M}} k_{56} C_{*}^{M} \\
= & \frac{k_{1} k_{2}+k_{1} k_{59} C_{*}^{M}+k_{2} k_{59} C_{*}+k_{1} k_{56} C_{*}^{M 2}+k_{2} k_{54} C_{*}^{2}}{C_{*} C_{*}^{M}}-k_{72} k_{54} C_{*}-k_{83} k_{56} C_{*}^{M}
\end{aligned}
$$

In Larsen (2016) [9], we found two cubic polynomials $P, P_{M}$ such that

$$
P(C)=0, \quad P_{M}\left(C_{M}\right)=0
$$

whenever $\left(C, C_{M}, G F, G I\right)$ is a nonnegative singular point of $f$. We shall need the following lemma.

Lemma 1 Assume $k_{72}=k_{54}, k_{83}=k_{56}$. Then

where

$$
P_{M}\left(C_{M}\right)=b_{2} C_{M}^{2}+b_{1} C_{M}+b_{0}
$$

$$
\begin{gathered}
b_{2}=-k_{56}^{2} k_{54}^{2} k_{95}\left(k_{1}+k_{2}\right) \\
b_{1}=k_{2} k_{54}^{2} k_{56} k_{95} k \\
b_{0}=-k_{2}^{2} k_{59} k_{54}^{2} k_{95}
\end{gathered}
$$

Proof. The coefficient to $C_{M}^{4}$ is according to Larsen (2016), [9]

$$
a_{M} a^{2}-f_{M} f a=0
$$

$a=-k_{56}^{2}, b=k_{56}\left(k_{95}-k_{59}-k_{2}\right), c=-k_{2} k_{59}, d=k_{2} k_{54}, f=k_{56} k_{54}$ and $a_{M}=-k_{54}^{2}, b_{M}=k_{54}\left(k_{95}-k_{59}-k_{1}\right)$, $c_{M}=-k_{1} k_{59}, d_{M}=k_{1} k_{56}, f_{M}=k_{56} k_{54}$. The coefficient to $C_{M}^{3}$ is according to Larsen (2016), [9]

$$
\begin{aligned}
- & d_{M} f^{2}-f_{M} b f+2 a_{M} a b+b_{M} a f-f_{M} a d \\
= & -k_{1} k_{56}^{3} k_{54}^{2}-k_{56}^{3} k_{54}^{2}\left(k_{95}-k_{59}-k_{2}\right)+2 k_{54}^{2} k_{56}^{3}\left(k_{95}-k_{59}-k_{2}\right) \\
& +k_{54}\left(k_{95}-k_{59}-k_{1}\right)\left(-k_{56}^{2}\right) k_{56} k_{54}+k_{56}^{3} k_{54}^{2} k_{2}
\end{aligned}
$$

Everything cancels out and leaves a zero. The coefficient to $C_{M}^{2}$ is according to Larsen (2016), [9]

$$
\begin{aligned}
a_{M} & b^{2}+c_{M} f^{2}+b_{M} a d-f_{M} b d+b_{M} b f+2 a_{M} a c-2 d_{M} d f-f_{M} c f \\
= & -k_{54}^{2} k_{56}^{2}\left(k_{95}-k_{59}-k_{2}\right)^{2}-k_{1} k_{59}\left(k_{56} k_{54}\right)^{2}+k_{54}\left(k_{95}-k_{59}-k_{1}\right)\left(-k_{56}^{2}\right) k_{2} k_{54} \\
& -k_{56}^{2} k_{54}\left(k_{95}-k_{59}-k_{2}\right) k_{2} k_{54}+k_{54}\left(k_{95}-k_{59}-k_{1}\right) k_{56}\left(k_{95}-k_{59}-k_{2}\right) k_{54} k_{56} \\
& +2\left(-k_{54}^{2}\right)\left(-k_{56}^{2}\right)\left(-k_{2} k_{59}\right)-k_{56} k_{54}\left(-k_{2} k_{59}\right) k_{56} k_{54}-2 k_{1} k_{2} k_{54}^{2} k_{56}^{2}
\end{aligned}
$$

Square $b^{2}$ and multiply $b_{M} b$ to get

$$
\begin{aligned}
= & -k_{54}^{2} k_{56}^{2}\left(\left(k_{95}-k_{59}\right)^{2}+k_{2}^{2}-2 k_{2}\left(k_{95}-k_{59}\right)\right)-k_{1} k_{59}\left(k_{56} k_{54}\right)^{2} \\
& +k_{54}\left(k_{95}-k_{59}-k_{1}\right)\left(-k_{56}^{2}\right) k_{2} k_{54}-k_{56}^{2} k_{54}\left(k_{95}-k_{59}-k_{2}\right) k_{2} k_{54} \\
& +k_{54}^{2} k_{56}^{2}\left(\left(k_{95}-k_{59}\right)^{2}-\left(k_{1}+k_{2}\right)\left(k_{95}-k_{59}\right)+k_{1} k_{2}\right) \\
& +2\left(-k_{54}^{2}\right)\left(-k_{56}^{2}\right)\left(-k_{2} k_{59}\right)-k_{56} k_{54}\left(-k_{2} k_{59}\right) k_{56} k_{54}-2 k_{1} k_{2} k_{54}^{2} k_{56}^{2}
\end{aligned}
$$


Everything cancels out except

$$
-k_{56}^{2} k_{54}^{2} k_{95}\left(k_{1}+k_{2}\right)
$$

The coefficient to $C_{M}$ is according to Larsen (2016), [9]

$$
\begin{aligned}
b_{M} c f+2 c_{M} d f-f_{M} c d-d_{M} d^{2}+2 a_{M} b c+b_{M} b d \\
=k_{54}\left(k_{95}-k_{59}-k_{1}\right)\left(-k_{2} k_{59}\right) k_{56} k_{54}+2\left(-k_{1} k_{59}\right) k_{2} k_{54}^{2} k_{56} \\
\quad+k_{54} k_{56} k_{2} k_{59} k_{2} k_{54}-k_{1} k_{56}\left(k_{2} k_{54}\right)^{2}+2\left(-k_{54}^{2}\right) k_{56}\left(k_{95}-k_{59}-k_{2}\right)\left(-k_{2} k_{59}\right) \\
\quad+k_{54}\left(k_{95}-k_{59}-k_{1}\right) k_{56}\left(k_{95}-k_{59}-k_{2}\right) k_{2} k_{54}
\end{aligned}
$$

Multiply

$$
b_{M} b d=k_{54}^{2} k_{56}\left(\left(k_{95}-k_{59}\right)^{2}-\left(k_{95}-k_{59}\right)\left(k_{1}+k_{2}\right)+k_{1} k_{2}\right) k_{2}
$$

Everything cancels out except

$$
\begin{aligned}
& =-k_{2} k_{56} k_{54}^{2} k_{95} k_{59}+k_{2} k_{54}^{2} k_{56} k_{95}^{2}-k_{2} k_{54}^{2} k_{56} k_{95} k_{1}-k_{2}^{2} k_{54}^{2} k_{56} k_{95} \\
& =k_{2} k_{54}^{2} k_{56} k_{95} k
\end{aligned}
$$

Finally the constant term is

$$
\begin{aligned}
& c_{M} d^{2}+a_{M} c^{2}+b_{M} c d \\
& =-k_{1} k_{59}\left(k_{2} k_{54}\right)^{2}-k_{54}^{2}\left(k_{2} k_{59}\right)^{2}+k_{54}\left(k_{95}-k_{59}-k_{1}\right)\left(-k_{2} k_{59}\right) k_{2} k_{54} \\
& =-k_{95} k_{54}^{2} k_{59} k_{2}^{2}
\end{aligned}
$$

The lemma follows.

Theorem 2 Assume $k_{72}=k_{54}, k_{83}=k_{56}$ When $k=k_{95}-k_{59}-k_{1}-k_{2}>0, \quad \nabla=k^{2}-4 k_{59}\left(k_{1}+k_{2}\right)>0$ there are exactly two positive singular points of $f$

$$
\left(C_{*}^{-}, C_{*}^{M-}, G F_{*}, G I_{*}^{-}\right),\left(C_{*}^{+}, C_{*}^{M+}, G F_{*}, G I_{*}^{+}\right)
$$

where

$$
C_{*}^{-}<C_{*}^{+}, \quad C_{*}^{M-}<C_{*}^{M+}
$$

Proof. We have

$$
P(C)=a_{2} C^{2}+a_{1} C+a_{0}, \quad P_{M}\left(C_{M}\right)=b_{2} C_{M}^{2}+b_{1} C_{M}+b_{0}
$$

where

$$
\begin{gathered}
a_{2}=-k_{56}^{2} k_{54}^{2} k_{95}\left(k_{1}+k_{2}\right) \\
a_{1}=k_{56}^{2} k_{54} k_{1} k_{95} k \\
a_{0}=-k_{1}^{2} k_{59} k_{56}^{2} k_{95}
\end{gathered}
$$

and

$$
\begin{gathered}
b_{2}=-k_{56}^{2} k_{54}^{2} k_{95}\left(k_{1}+k_{2}\right) \\
b_{1}=k_{56} k_{54}^{2} k_{2} k_{95} k \\
b_{0}=-k_{2}^{2} k_{59} k_{54}^{2} k_{95}
\end{gathered}
$$


due to symmetry of $P, P_{M}$. When $k>0, \nabla>0, P$ and $P_{M}$ have two positive roots

$$
C_{*}^{-}<C_{*}^{+}
$$

in $P$ and

$$
C_{*}^{M-}<C_{*}^{M+}
$$

in $P_{M}$, see (15) and (16) below. We are going to verify that

$$
\left(C_{*}^{-}, C_{*}^{M-}, G F_{*}, G I_{*}^{-}\right),\left(C_{*}^{+}, C_{*}^{M+}, G F_{*}, G I_{*}^{+}\right)
$$

are singular points of $f$ and that

$$
\left(C_{*}^{-}, C_{*}^{M+}, G F_{*}, G I_{*}^{-,+}\right),\left(C_{*}^{+}, C_{*}^{M-}, G F_{*}, G I_{*}^{+,-}\right)
$$

are not singular points of $f$. Here

$$
G I_{*}^{-,+}=\frac{k_{95}}{k_{54} C_{*}^{-}+k_{56} C_{*}^{M+}+k_{59}}
$$

and

$$
G I_{*}^{+,-}=\frac{k_{95}}{k_{54} C_{*}^{+}+k_{56} C_{*}^{M-}+k_{59}}
$$

Also

$$
\begin{aligned}
& G I_{*}^{+}=\frac{k_{95}}{k_{54} C_{*}^{+}+k_{56} C_{*}^{M+}+k_{59}} \\
& G I_{*}^{-}=\frac{k_{95}}{k_{54} C_{*}^{-}+k_{56} C_{*}^{M-}+k_{59}}
\end{aligned}
$$

We have

$$
C_{*}^{ \pm}=k_{1} \frac{k \pm \sqrt{\nabla}}{2 k_{54}\left(k_{1}+k_{2}\right)}
$$

and logically equivalent

$$
C_{*}^{M \pm}=k_{2} \frac{k \pm \sqrt{\nabla}}{2 k_{56}\left(k_{1}+k_{2}\right)}
$$

where $\nabla=k^{2}-4 k_{59}\left(k_{1}+k_{2}\right)>0$. To see (15) compute

$$
\begin{aligned}
\tilde{\nabla} & =a_{1}^{2}-4 a_{0} a_{2} \\
& =k_{56}^{4} k_{54}^{2} k_{1}^{2} k_{95}^{2} k^{2}-4 k_{1}^{2} k_{59} k_{56}^{2} k_{95} \cdot k_{56}^{2} k_{54}^{2} k_{95}\left(k_{1}+k_{2}\right) \\
& =k_{56}^{4} k_{54}^{2} k_{1}^{2} k_{95}^{2}\left(k^{2}-4 k_{59}\left(k_{1}+k_{2}\right)\right)
\end{aligned}
$$

So

$$
C_{*}^{ \pm}=\frac{-k_{56}^{2} k_{54} k_{1} k_{95} k \pm k_{56}^{2} k_{54} k_{1} k_{95} \sqrt{\nabla}}{-2 k_{56}^{2} k_{54}^{2} k_{95}\left(k_{1}+k_{2}\right)}
$$

and from this the formula follows. And (16) is a similar computation. 
We shall insert (15), (16) in the first coordinate of $f$, multiplied with $\left(k_{54} C_{*}^{ \pm}+k_{56} C_{*}^{M \pm}+k_{59}\right)>0$

$$
\begin{aligned}
& \left(k_{54} C_{*}^{ \pm}+k_{1}\right)\left(k_{54} C_{*}^{ \pm}+k_{56} C_{*}^{M \pm}+k_{59}\right)-k_{54} k_{95} C_{*}^{ \pm} \\
& =\left(k_{1} \frac{k \pm \sqrt{\nabla}}{2\left(k_{1}+k_{2}\right)}+k_{1}\right)\left(k_{1} \frac{k \pm \sqrt{\nabla}}{2\left(k_{1}+k_{2}\right)}+k_{2} \frac{k \pm \sqrt{\nabla}}{2\left(k_{1}+k_{2}\right)}+k_{59}\right)-k_{95} k_{1} \frac{k \pm \sqrt{\nabla}}{2\left(k_{1}+k_{2}\right)}=0
\end{aligned}
$$

Now abbreviate $x=k \pm \sqrt{\nabla}$ and find

$$
=\left(k_{1} \frac{x}{2\left(k_{1}+k_{2}\right)}+k_{1}\right)\left(\frac{x}{2}+k_{59}\right)-k_{95} k_{1} \frac{x}{2\left(k_{1}+k_{2}\right)}=0
$$

Multiply with $2\left(k_{1}+k_{2}\right)>0$ to get

$$
\left(k_{1} x+2\left(k_{1}+k_{2}\right) k_{1}\right)\left(\frac{x}{2}+k_{59}\right)-k_{95} k_{1} x=0
$$

But this amounts to

$$
=k_{1}\left(\frac{1}{2} x^{2}-k x+2 k_{59}\left(k_{1}+k_{2}\right)\right)
$$

and this vanishes due to the formula for roots of quadratic polynomials. That the second coordinate vanishes is logically equivalent. So (11) are singular points of $f$.

We shall now argue, that

$$
\left(C_{*}^{-}, C_{*}^{M+}, G F_{*}, G I_{*}^{-,+}\right)
$$

is not a singular point of $f$. To this end define

$$
y=k+\sqrt{\nabla}, \quad x=k-\sqrt{\nabla}
$$

Insert the formulas (15), (16) for $C_{*}^{-}, C_{*}^{M+}$ in the first coordinate of $f$ multiplied with $k_{54} C_{*}^{-}+k_{56} C_{*}^{M+}+k_{59}$ to get

$$
\left(k_{1} \frac{x}{2\left(k_{1}+k_{2}\right)}+k_{1}\right)\left(k_{1} \frac{x}{2\left(k_{1}+k_{2}\right)}+k_{2} \frac{(y-x)+x}{2\left(k_{1}+k_{2}\right)}+k_{59}\right)-k_{95} k_{1} \frac{x}{2\left(k_{1}+k_{2}\right)}
$$

Multiply with $2\left(k_{1}+k_{2}\right)$ to find

$$
\begin{aligned}
& k_{1}\left(\left(x+2\left(k_{1}+k_{2}\right)\right)\left(\frac{x}{2}+k_{59}\right)-k_{95} x\right) \\
& +\left(k_{1} \frac{x}{2\left(k_{1}+k_{2}\right)}+k_{1}\right) k_{2}(y-x)
\end{aligned}
$$

But (17) is zero by the above and (18) is nonzero. So $\left(C_{*}^{-}, C_{*}^{M+}, G F_{*}, G I_{*}^{-,+}\right)$is not a singular point. That $\left(C_{*}^{+}, C_{*}^{M-}, G F_{*}, G I_{*}^{+,-}\right)$is not a singular of $f$ is logically equivalent. The theorem follows.

In the remainder of the proof of Theorem 1 , we assume, that

$$
k_{72}=k_{54}, \quad k_{83}=k_{56}, \quad k>0, \quad \nabla>0
$$

We shall now verify that $\left(C_{*}^{+}, C_{*}^{M+}, G F_{*}, G I_{*}^{+}\right)$is unstable. We shall show that $\operatorname{det} \tilde{B}>0$.

But we have

$$
\operatorname{det} \tilde{B}=\frac{k_{1} k_{56}^{2}\left(C_{*}^{M+}\right)^{2}+k_{2} k_{54}^{2}\left(C_{*}^{+}\right)^{2}-k_{1} k_{2} k_{59}}{C_{*}^{+} C_{*}^{M+}}
$$


Simply insert (15) and (16) in the numerator

$$
\begin{aligned}
& k_{1} k_{2}^{2}\left(\frac{k+\sqrt{\nabla}}{2\left(k_{1}+k_{2}\right)}\right)^{2}+k_{1}^{2} k_{2}\left(\frac{k+\sqrt{\nabla}}{2\left(k_{1}+k_{2}\right)}\right)^{2}-k_{1} k_{2} k_{59} \\
& =k_{1} k_{2}\left(\left(k_{1}+k_{2}\right)\left(\frac{k+\sqrt{\nabla}}{2\left(k_{1}+k_{2}\right)}\right)^{2}-k_{59}\right) \\
& =k_{1} k_{2}\left(\frac{1}{4\left(k_{1}+k_{2}\right)}\left(k^{2}+\nabla+2 k \sqrt{\nabla}\right)-k_{59}\right) \\
& =\frac{k_{1} k_{2}}{4\left(k_{1}+k_{2}\right)}\left(k^{2}+\nabla+2 k \sqrt{\nabla}-4 k_{59}\left(k_{1}+k_{2}\right)\right)
\end{aligned}
$$

Now we use that

$$
k^{2}+\nabla-4 k_{59}\left(k_{1}+k_{2}\right)=2 \nabla
$$

so

$$
2 \nabla+2 k \sqrt{\nabla}>0
$$

is equivalent to

$$
\nabla>-k \sqrt{\nabla}
$$

The right hand side here is negative and the left hand side is positive. Thus $\tilde{B}$ has a positive eigenvalue. So $\left(C_{*}^{+}, C_{*}^{M+}, G F_{*}, G I_{*}^{+}\right)$is unstable.

We shall now show that $\left(C_{*}^{-}, C_{*}^{M-}, G F_{*}, G I_{*}^{-}\right)$is stable, when $k_{59}$ is small. We shall use the Routh Hurwitz criterion. So we start by showing, that $\operatorname{det} \tilde{B}<0$. But similarly to the above

$$
\begin{aligned}
\text { numerator }(\operatorname{det} \tilde{B}) & =k_{1} k_{2}^{2}\left(\frac{k-\sqrt{\nabla}}{2\left(k_{1}+k_{2}\right)}\right)^{2}+k_{1}^{2} k_{2}\left(\frac{k-\sqrt{\nabla}}{2\left(k_{1}+k_{2}\right)}\right)^{2}-k_{1} k_{2} k_{59} \\
& =\frac{k_{1} k_{2}}{4\left(k_{1}+k_{2}\right)}\left(k^{2}+\nabla-2 k \sqrt{\nabla}-4 k_{59}\left(k_{1}+k_{2}\right)\right) \\
& =\frac{k_{1} k_{2}}{4\left(k_{1}+k_{2}\right)}(2 \nabla-2 k \sqrt{\nabla})<0
\end{aligned}
$$

But this amounts to

$$
\nabla<k \sqrt{\nabla}
$$

which is equivalent to

$$
\nabla^{2}<k^{2} \nabla
$$

and this again is equivalent to

$$
\nabla<k^{2}
$$

and from this it follows that $\operatorname{det} \tilde{B}<0$. We have the following formula for $\tau$

$$
\begin{aligned}
\tau= & \frac{1}{C_{*}^{-} C_{*}^{M-}}\left(k_{1} k_{2}+k_{1} k_{59} C_{*}^{M-}+k_{2} k_{59} C_{*}^{-}+k_{1} k_{56}\left(C_{*}^{M-}\right)^{2}+k_{2} k_{54}\left(C_{*}^{-}\right)^{2}\right. \\
& \left.-k_{54}^{2}\left(C_{*}^{-}\right)^{2} C_{*}^{M-}-k_{56}^{2} C_{*}^{-}\left(C_{*}^{M-}\right)^{2}\right)
\end{aligned}
$$


And a formula for $\sigma$

$$
\sigma=-\frac{k_{1}}{C_{*}^{-}}-\frac{k_{2}}{C_{*}^{M-}}-k_{59}-k_{54} C_{*}^{-}-k_{56} C_{*}^{M-}
$$

Define

$$
x=\frac{k-\sqrt{\nabla}}{2\left(k_{1}+k_{2}\right)}
$$

so that

$$
C_{*}^{-}=\frac{k_{1}}{k_{54}} x, \quad C_{*}^{M-}=\frac{k_{2}}{k_{56}} x
$$

Now introduce these two formulas in the formulas for $\sigma, \tau$

$$
\begin{gathered}
\sigma=-\frac{k_{54}}{x}-\frac{k_{56}}{x}-k_{59}-k_{1} x-k_{2} x \\
\tau=\frac{k_{1} k_{2}+k_{1} k_{59} k_{2} \frac{x}{k_{56}}+k_{2} k_{59} k_{1} \frac{x}{k_{54}}+k_{1} k_{2}^{2} \frac{x^{2}}{k_{56}}+k_{1}^{2} k_{2} \frac{x^{2}}{k_{54}}-\frac{k_{1}^{2} k_{2}}{k_{56}} x^{3}-\frac{k_{1} k_{2}^{2}}{k_{54}} x^{3}}{\frac{1}{k_{54} k_{56}} k_{1} k_{2} x^{2}}
\end{gathered}
$$

Notice that $\tau>0$ for small $x>0$. Also

$$
\delta=\frac{-k_{59}+k_{1} x^{2}+k_{2} x^{2}}{\frac{1}{k_{54} k_{56}} x^{2}}
$$

is negative for small $x>0$. The Routh Hurwitz criterion says in our framework, that $\sigma<0, \tau>0, \delta<0$, $-\sigma \tau+\delta>0$ is equivalent to stability of $\left(C_{*}^{-}, C_{*}^{M-}, G F_{*}, G I_{*}^{-}\right)$. But $-\sigma \tau+\delta>0$ is equivalent to $x^{3}(-\sigma \tau+\delta)>0$ because our assumptions imply $x>0$. So $x^{3}(-\sigma \tau+\delta)>0$ is equivalent to

$$
\begin{aligned}
& \left(k_{54}+k_{56}+k_{59} x+k_{1} x^{2}+k_{2} x^{2}\right)\left(1+\left(\frac{x}{k_{56}}+\frac{x}{k_{54}}\right) k_{59}+k_{2} \frac{x^{2}}{k_{56}}+k_{1} \frac{x^{2}}{k_{54}}-k_{1} \frac{x^{3}}{k_{56}}-k_{2} \frac{x^{3}}{k_{54}}\right) k_{54} k_{56} \\
& +x\left(-k_{59}+k_{1} x^{2}+k_{2} x^{2}\right) k_{56} k_{54}>0
\end{aligned}
$$

This equation holds for small $k_{59}$. So $\left(C_{*}^{-}, C_{*}^{M-}, G F_{*}, G I_{*}^{-}\right)$is stable for small $k_{59}$. This follows by writing

$$
1-\sqrt{1+z}=-\int_{0}^{1} \frac{d}{d s} \sqrt{1+z s} \mathrm{~d} s=-z \int_{0}^{1} \frac{1}{2 \sqrt{1+z s}} \mathrm{~d} s=z h(z)
$$

where $z \in]-1,1[$ and $h$ is smooth. This is the standard trick from singularity theory. Then

$$
\begin{aligned}
x & =\frac{k\left(1-\sqrt{1-\frac{4 k_{59}\left(k_{1}+k_{2}\right)}{k^{2}}}\right)}{2\left(k_{1}+k_{2}\right)} \\
& =\frac{k}{2\left(k_{1}+k_{2}\right)} \frac{-4 k_{59}\left(k_{1}+k_{2}\right)}{k^{2}} h\left(-\frac{4 k_{59}\left(k_{1}+k_{2}\right)}{k^{2}}\right) \\
& =-2 \frac{k_{59}}{k} h\left(-\frac{4 k_{59}\left(k_{1}+k_{2}\right)}{k^{2}}\right)
\end{aligned}
$$


And from this it follows that $\left(C_{*}^{-}, C_{*}^{M-}, G F_{*}, G I_{*}^{-}\right)$is stable for small $k_{59}$. To be precise, given $k_{95}=k_{95}^{0}$, $k_{1}=k_{1}^{0}, k_{2}=k_{2}^{0}, k_{59}=k_{59}^{0} \in \mathbb{R}_{+}$, such that $k=k_{0}=k_{95}^{0}-k_{59}^{0}-k_{1}^{0}-k_{2}^{0}>0$ and, $\nabla^{0}=k_{0}^{2}-4 k_{59}^{0}\left(k_{1}^{0}+k_{2}^{0}\right)>0$, then there exists $\varepsilon>0, \varepsilon<k_{59}^{0}$ such that $c_{*}^{-}$is stable when $k_{59}<\varepsilon$. Theorem 2 follows.

Consider the mass action kinetic system in the species $C, G F, G I, P$ cancer cells, growth factor, growth inhibitor and a protein, respectively.

$$
\begin{aligned}
& G F \rightarrow C \\
& C+G I \rightarrow 0 \\
& C \rightarrow 2 C \\
& 0 \leftrightarrows G F \\
& 0 \leftrightarrows G I \\
& 0 \leftrightarrows C \rightarrow P \\
& 2 C+P \rightarrow 3 C
\end{aligned}
$$

The complexes are $C(1)=G F, C(2)=C, C(3)=G I+C, C(4)=0, C(5)=2 C, C(6)=G I, \quad C(7)=P$, $C(8)=2 C+P, C(9)=3 C$. And this defines the rate constants $k_{i j}$. With mass action kinetics the ODE $\mathrm{s}$ become

$$
\begin{aligned}
& C^{\prime}=k_{21} G F-k_{43} C \cdot G I+k_{52} C-\left(k_{42}+k_{72}\right) C+k_{24}+k_{98} C^{2} \cdot P \\
& G F^{\prime}=-\left(k_{21}+k_{41}\right) G F+k_{14} \\
& G I^{\prime}=-k_{43} C \cdot G I+k_{64}-k_{46} G I \\
& P^{\prime}=k_{72} C-k_{98} C^{2} \cdot P
\end{aligned}
$$

see Horn and Jackson (1972), [10]. Notice that (24), (25) are the Brusselator, which is known to have oscillating solutions for some values of the parameters, see Sarmah et al. (2015), [11]. Subtracting $2 C$ on both sides of (25) gives the reaction $P \rightarrow C$. Let $k_{21}=0.01, k_{43}=0.01, k_{52}=0.01, k_{72}=2, k_{98}=0.9, \quad k_{46}=0.01, k_{64}=0.01$, $k_{24}=1, k_{42}=1, k_{14}=0.01, k_{41}=0.01$. With these parameter values and initial conditions $\left(C_{0}, G F_{0}, G I_{0}, P_{0}\right)=$ $(1,1,1,1)$ the system oscillates, see Figure 1.

\section{Eigenvalues with Negative Real Part}

In this section $\mu_{F}=\mu_{I}=0, g=0$ in the discrete model $T$ of the introduction. The purpose of this section is to find a formula for the rate of change of cancer growth

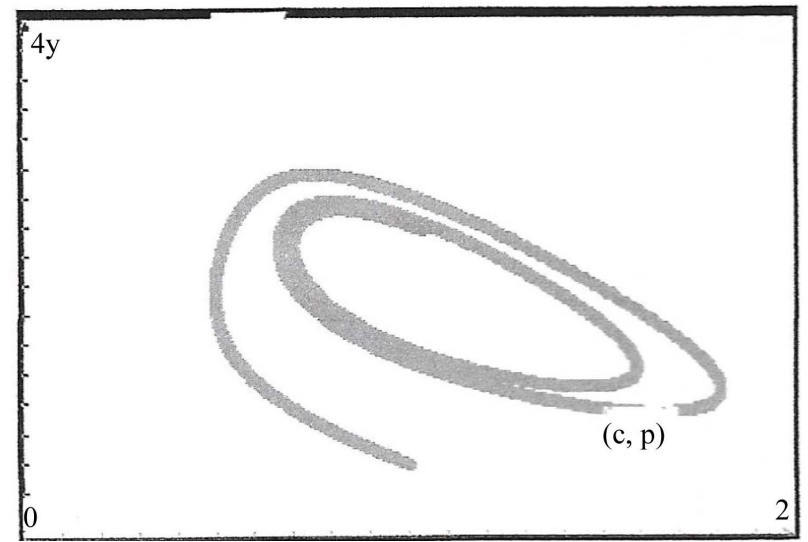

Figure 1. The oscillating mass action kinetic system. I have plotted $\mathrm{P}$ versus $\mathrm{C}$. 


$$
\frac{\mathrm{d} C}{\mathrm{~d} t}(0)
$$

on the hyperplane $C=0$. Here $(C(t), G F(t), G I(t))$ is an integral curve of the vector field $Y$, defined below. There are four cases to consider. First assume, that $\nabla<0$. Let $\lambda_{+}=a+i b=\lambda_{1}+i \lambda_{2}$. We shall assume that $a^{2}-b^{2}>0$. Define

$$
U=\left(\begin{array}{ccc}
0 & 1-a & -b \\
\beta & -\delta & 0 \\
-\alpha & -\sigma & 0
\end{array}\right)
$$

and compute, when $\operatorname{det} U \neq 0$

$$
U^{-1}=\frac{1}{\operatorname{det} U}\left(\begin{array}{ccc}
0 & \sigma b & -\delta b \\
0 & -\alpha b & -\beta b \\
-(\alpha \delta+\beta \sigma) & -\alpha(1-a) & -\beta(1-a)
\end{array}\right)
$$

If $\lambda_{+}$has negative real part we might be able to find an affine vector field whose time one map is $T^{2}$. Notice that

$$
T^{2}(y)=A^{2} y+A c+c
$$

By Larsen (2016), [1],

$$
U^{-1} A U=\left(\begin{array}{ccc}
1 & 0 & 0 \\
0 & a & b \\
0 & -b & a
\end{array}\right)
$$

Then

$$
U^{-1} A^{2} U=\left(\begin{array}{ccc}
1 & 0 & 0 \\
0 & a^{2}-b^{2} & 2 a b \\
0 & -2 a b & a^{2}-b^{2}
\end{array}\right)
$$

Define the vector field

$$
X(z)=\left(\begin{array}{rrr}
0 & 0 & 0 \\
0 & a_{2} & b_{2} \\
0 & -b_{2} & a_{2}
\end{array}\right) z+d
$$

$d, z \in \mathbb{R}^{3}$ and let

$$
L_{2}=\left(\begin{array}{cc}
a_{2} & b_{2} \\
-b_{2} & a_{2}
\end{array}\right)
$$

where $a_{2}, b_{2} \in \mathbb{R}$. The flow of $X$ is

$$
\begin{aligned}
& \Phi^{X}(t, w)_{1}=x_{1}+t d_{1} \\
& \Phi^{X}(t, w)_{2,3}=\exp \left(L_{2} t\right) x+L_{2}^{-1}\left(\exp \left(L_{2} t\right)-\mathrm{id}\right) d
\end{aligned}
$$

where $w=\left(x_{1}, x_{2}, x_{3}\right) \in \mathbb{R}^{3}, x=\left(x_{2}, x_{3}\right), d=\left(d_{2}, d_{3}\right) \in \mathbb{R}^{2}, x_{1}, d_{1} \in \mathbb{R}$. Also

$$
\exp \left(L_{2} t\right)=\left(\begin{array}{cc}
\mathrm{e}^{a_{2} t} \cos b_{2} t & \mathrm{e}^{a_{2} t} \sin b_{2} t \\
-\mathrm{e}^{a_{2} t} \sin b_{2} t & \mathrm{e}^{a_{2} t} \cos b_{2} t
\end{array}\right)
$$


If

$$
\begin{gathered}
\mathrm{e}^{a_{2}} \cos b_{2}=a^{2}-b^{2} \\
\mathrm{e}^{a_{2}} \sin b_{2}=2 a b
\end{gathered}
$$

then

$$
\exp \left(L_{2}\right)=\left(\begin{array}{cc}
a^{2}-b^{2} & 2 a b \\
-2 a b & a^{2}-b^{2}
\end{array}\right)
$$

Assume that $a^{2}>b^{2}$. Then we can let

$$
b_{2}=\tan ^{-1}\left(\frac{2 a b}{a^{2}-b^{2}}\right)
$$

But this means that

$$
\sin b_{2}=\frac{2 a b}{a^{2}+b^{2}}
$$

because we have

$$
\sin \left(\tan ^{-1}(x)\right)=\frac{x}{\sqrt{1+x^{2}}}
$$

$x \in]-\frac{\pi}{2}, \frac{\pi}{2}[$. So we get

$$
\mathrm{e}^{a_{2}}=a^{2}+b^{2}
$$

i.e. $a_{2}=\ln \left(a^{2}+b^{2}\right)$. Consider first the immune therapy model

$$
c=\left(\begin{array}{c}
0 \\
0 \\
c_{3}
\end{array}\right), c_{3}>0
$$

So assuming $\operatorname{det} U \neq 0$

$$
U^{-1}(A c+c)=\frac{1}{\operatorname{det} U}\left(\begin{array}{c}
-\delta \lambda_{2} 2 c_{3} \\
-\beta \lambda_{2} 2 c_{3} \\
-\beta c_{3}(\alpha \delta+\beta \sigma)-2 \beta c_{3}\left(1-\lambda_{1}\right)
\end{array}\right)
$$

We want to have

$$
d_{1}=U^{-1}(A c+c)
$$

and

$$
L_{2}^{-1}\left(\exp \left(L_{2}\right)-\mathrm{id}\right)\left(\begin{array}{l}
d_{2} \\
d_{3}
\end{array}\right)=\left(U^{-1}(A c+c)\right)_{2,3}
$$

such that

$$
\Phi_{1}^{X}(w)=U^{-1} A^{2} U(w)+U^{-1}(A c+c)
$$

Here $\Phi_{1}^{X}$ denotes the time one map of $X$ and $w \in \mathbb{R}^{3}$. Define 
Then

$$
Y(w)=D U \circ X \circ U^{-1}(w)
$$

$$
\Phi^{Y}(t, w)=U \circ \Phi^{X}\left(t, U^{-1} w\right)
$$

Thus

$$
\Phi_{1}^{Y}(w)=A^{2} w+A c+c=T^{2}(w)
$$

Now

$$
\left(\begin{array}{l}
d_{2} \\
d_{3}
\end{array}\right)=\frac{1}{\operatorname{det} U}\left(\begin{array}{cc}
a^{2}-b^{2}-1 & 2 a b \\
-2 a b & a^{2}-b^{2}-1
\end{array}\right)^{-1} L_{2}\left(\begin{array}{c}
-2 \beta \lambda_{2} c_{3} \\
-2 \beta c_{3}\left(1-\lambda_{1}\right)-\beta c_{3}(\alpha \delta+\beta \sigma)
\end{array}\right)
$$

Define

$$
p=\left(a^{2}-b^{2}-1\right)^{2}+4 a^{2} b^{2}
$$

Let $U_{1}$ denote the first row in $U$. Compute letting

$$
\begin{aligned}
x=U^{-1}\left(\begin{array}{c}
0 \\
G F_{0} \\
G I_{0}
\end{array}\right) \\
\frac{\mathrm{d} C}{\mathrm{~d} t}(0)=\frac{\mathrm{d}}{\mathrm{d} t}\left(U_{1} \circ \Phi^{X}\right)(0, x)=-\frac{b_{2}}{\operatorname{det} U}\left(\alpha G F_{0}+\beta G I_{0}\right)\left(\left(1-\lambda_{1}\right)^{2}+\lambda_{2}^{2}\right) \\
+\frac{\left(1-\lambda_{1}\right)^{2}+\lambda_{2}^{2}}{p \operatorname{det} U}\left(b_{2}\left(a^{2}-b^{2}-1\right)-2 a b a_{2}\right)\left(-2 \beta c_{3}\right) \\
+\frac{1}{p \operatorname{det} U}\left(1-\lambda_{1},-\lambda_{2}\right)\left(\begin{array}{l}
b_{2}\left(a^{2}-b^{2}-1\right)-2 a b a_{2} \\
2 a b b_{2}+\left(a^{2}-b^{2}-1\right) a_{2}
\end{array}\right)\left(-\beta c_{3}\right)(\alpha \delta+\beta \sigma)
\end{aligned}
$$

where $(C, G F, G I)(t)$ is an integral curve of $Y$ through $\left(0, G F_{0}, G I_{0}\right) \in \mathbb{R}^{3}$. And, because $\left(1-\lambda_{1}\right)^{2}+\lambda_{2}^{2}=$ $-(\alpha \delta+\beta \sigma)$ this is equal to

$$
\begin{aligned}
\frac{\mathrm{d} C}{\mathrm{~d} t}(0)= & -\frac{b_{2}}{\operatorname{det} U}\left(\alpha G F_{0}+\beta G I_{0}\right)(-(\alpha \delta+\beta \sigma)) \\
& +\frac{1}{p \operatorname{det} U}\left(\left(b_{2}\left(a^{2}-b^{2}-1\right)-2 a b a_{2}\right)\left(2 \beta c_{3}\right)(\alpha \delta+\beta \sigma)\right. \\
& +(1-a)\left(b_{2}\left(a^{2}-b^{2}-1\right)-2 a b a_{2}\right)(-(\alpha \delta+\beta \sigma)) \beta c_{3} \\
& \left.+b\left(2 a b b_{2}+a_{2}\left(a^{2}-b^{2}-1\right)\right)(\alpha \delta+\beta \sigma) \beta c_{3}\right)
\end{aligned}
$$

Now suppose $\nabla>0, \lambda_{+}, \lambda_{-} \neq 0, \alpha \delta+\beta \sigma<0$ and $1, \lambda_{+}, \lambda_{-}$distinct and define

$$
D=\left(\begin{array}{ccc}
0 & 1-\lambda_{+} & 1-\lambda_{-} \\
\beta & -\delta & -\delta \\
-\alpha & -\sigma & -\sigma
\end{array}\right)
$$

Then

$$
D^{-1} A D=\left(\begin{array}{ccc}
1 & 0 & 0 \\
0 & \lambda_{+} & 0 \\
0 & 0 & \lambda_{-}
\end{array}\right)
$$


when $\operatorname{det} D \neq 0$, because the columns of $D$ are eigenvectors of $A$ corresponding to eigenvalues $1, \lambda_{+}, \lambda_{-}$ respectively. Compute, when $\operatorname{det} D \neq 0$, the inverse

$$
D^{-1}=\frac{1}{\operatorname{det} D}\left(\begin{array}{ccc}
0 & -\sigma\left(\lambda_{+}-\lambda_{-}\right) & \delta\left(\lambda_{+}-\lambda_{-}\right) \\
(\beta \sigma+\alpha \delta) & \alpha\left(1-\lambda_{-}\right) & \beta\left(1-\lambda_{-}\right) \\
-(\beta \sigma+\alpha \delta) & -\alpha\left(1-\lambda_{+}\right) & -\beta\left(1-\lambda_{+}\right)
\end{array}\right)
$$

Then

$$
D^{-1}(A c+c)=D^{-1}\left(\begin{array}{c}
\beta c_{3} \\
0 \\
2 c_{3}
\end{array}\right)=\frac{1}{\operatorname{det} D}\left(\begin{array}{c}
2 c_{3} \delta\left(\lambda_{+}-\lambda_{-}\right) \\
\beta c_{3}(\alpha \delta+\beta \sigma)+2 c_{3} \beta\left(1-\lambda_{-}\right) \\
-\beta c_{3}(\alpha \delta+\beta \sigma)-2 c_{3} \beta\left(1-\lambda_{+}\right)
\end{array}\right)
$$

Define the vector field

$$
X(z)=\left(\begin{array}{ccc}
0 & 0 & 0 \\
0 & \ln \lambda_{+}^{2} & 0 \\
0 & 0 & \ln \lambda_{-}^{2}
\end{array}\right) z+d
$$

$z, d \in \mathbb{R}^{3} . X$ has flow

$$
\Phi^{X}(t, z)=\left(\begin{array}{ccc}
1 & 0 & 0 \\
0 & \exp \left(t \ln \lambda_{+}^{2}\right) & 0 \\
0 & 0 & \exp \left(t \ln \lambda_{-}^{2}\right)
\end{array}\right) z+\left(\begin{array}{c}
d_{1} t \\
\frac{1}{\ln \lambda_{+}^{2}}\left(\exp \left(t\left(\ln \lambda_{+}^{2}\right)\right)-1\right) d_{2} \\
\frac{1}{\ln \lambda_{-}^{2}}\left(\exp \left(t\left(\ln \lambda_{-}^{2}\right)\right)-1\right) d_{3}
\end{array}\right)
$$

and the time one map is

$$
\Phi_{1}^{X}(z)=D^{-1} A^{2} D z+\left(\begin{array}{c}
d_{1} \\
\frac{\lambda_{+}^{2}-1}{\ln \lambda_{+}^{2}} d_{2} \\
\frac{\lambda_{-}^{2}-1}{\ln \lambda_{-}^{2}} d_{3}
\end{array}\right)
$$

and we want this to be

$$
\Phi_{1}^{X}(z)=D^{-1} A^{2} D z+D^{-1}(A c+c)
$$

Then define the vector field

$$
Y(z)=D X D^{-1}(z)
$$

This vector field has time one map

$$
\Phi_{1}^{Y}(z)=A^{2} z+A c+c=T^{2}(z)
$$

Then arguing as before

$$
\frac{\left(\lambda_{+}^{2}-1\right) d_{2}}{\ln \left(\lambda_{+}\right)^{2}}=\frac{1}{\operatorname{det} D}\left(\beta c_{3}(\alpha \delta+\beta \sigma)+2 c_{3} \beta\left(1-\lambda_{-}\right)\right)
$$

and

$$
\frac{\left(\lambda_{-}^{2}-1\right) d_{3}}{\ln \left(\lambda_{-}\right)^{2}}=\frac{1}{\operatorname{det} D}\left(-\beta c_{3}(\alpha \delta+\beta \sigma)-2 c_{3} \beta\left(1-\lambda_{+}\right)\right)
$$

We can now find 


$$
\begin{aligned}
\frac{\mathrm{d} C}{\mathrm{~d} t}(0)= & \frac{\mathrm{d}}{\mathrm{d} t}\left(D_{1} \circ \Phi^{X}\right)(0, x) \\
= & \frac{1}{\operatorname{det} D}\left(\alpha G F_{0}+\beta G I_{0}\right)(-(\beta \sigma+\alpha \delta))\left(\ln \lambda_{+}^{2}-\ln \lambda_{-}^{2}\right) \\
& -\frac{\ln \lambda_{+}^{2}}{\lambda_{+}+1} \frac{1}{\operatorname{det} D}\left(\beta c_{3}(\alpha \delta+\beta \sigma)+2 \beta c_{3}\left(1-\lambda_{1}\right)\right) \\
& +\frac{\ln \lambda_{-}^{2}}{\lambda_{-}+1} \frac{1}{\operatorname{det} D}\left(\beta c_{3}(\alpha \delta+\beta \sigma)+2 \beta c_{3}\left(1-\lambda_{1}\right)\right)
\end{aligned}
$$

Next consider the chemo therapy model

$$
C=\left(\begin{array}{c}
c_{1} \\
0 \\
0
\end{array}\right), \quad c_{1}<0
$$

and initially, that $\nabla<0, \operatorname{det} U \neq 0, a^{2}-b^{2}>0$. Define the vector field $X$ by (26). It has flow (27), (28). Define the vector field

$$
Y=D U \circ X \circ U^{-1}
$$

We want this vector field to have time one map

$$
\Phi_{1}^{Y}(z)=A^{2} z+A c+c=T^{2}(z)
$$

Then we find

$$
U^{-1}(A c+c)=\frac{1}{\operatorname{det} U}\left(\begin{array}{c}
0 \\
-b c_{1}(\alpha \delta+\beta \sigma) \\
-(\alpha \delta+\beta \sigma)(2+\gamma) c_{1}-(1-a) c_{1}(\alpha \delta+\beta \sigma)
\end{array}\right)
$$

Now compute arguing as above

$$
\begin{aligned}
\left(\begin{array}{l}
d_{2} \\
d_{3}
\end{array}\right)= & \left(\begin{array}{cc}
a^{2}-b^{2}-1 & 2 a b \\
-2 a b & a^{2}-b^{2}-1
\end{array}\right)^{-1} L_{2}\left(U^{-1}(A c+c)_{2,3}\right) \\
= & \frac{1}{p \operatorname{det} U}\left(\begin{array}{cc}
a^{2}-b^{2}-1 & -2 a b \\
2 a b & a^{2}-b^{2}-1
\end{array}\right)\left(\begin{array}{cc}
a_{2} & b_{2} \\
-b_{2} & a_{2}
\end{array}\right) \\
& \cdot\left(\begin{array}{c}
-b c_{1}(\alpha \delta+\beta \sigma) \\
-(\alpha \delta+\beta \sigma)(2+\gamma) c_{1}-(1-a) c_{1}(\alpha \delta+\beta \sigma)
\end{array}\right)
\end{aligned}
$$

Finally we can find

$$
\begin{aligned}
\frac{\mathrm{d} C}{\mathrm{~d} t}(0)= & \frac{\mathrm{d}}{\mathrm{d} t}\left(U_{1} \circ \Phi^{X}\right)(0, x) \\
= & -\frac{b_{2}}{\operatorname{det} U}(-(\alpha \delta+\beta \sigma))\left(\alpha G F_{0}+\beta G I_{0}\right)+\left(1-\lambda_{1}\right) d_{2}-\lambda_{2} d_{3} \\
= & -\frac{b_{2}}{\operatorname{det} U}(-(\alpha \delta+\beta \sigma))\left(\alpha G F_{0}+\beta G I_{0}\right) \\
& +\frac{1}{p \operatorname{det} U}\left(-\left(1-\lambda_{1}\right)(\alpha \delta+\beta \sigma)(2+\gamma) c_{1}\left(\left(a^{2}-b^{2}-1\right) b_{2}-2 a b a_{2}\right)\right. \\
& +b(\alpha \delta+\beta \sigma)(2+\gamma) c_{1}\left(2 a b b_{2}+a_{2}\left(a^{2}-b^{2}-1\right)\right) \\
& +b^{2} c_{1}(-(\alpha \delta+\beta \sigma))\left(\left(a^{2}-b^{2}-1\right) b_{2}-2 a b a_{2}\right) \\
& \left.+\left(1-\lambda_{1}\right)^{2} c_{1}\left(b_{2}\left(a^{2}-b^{2}-1\right) b_{2}-2 a b a_{2}\right)(-(\alpha \delta+\beta \sigma))\right)
\end{aligned}
$$


and this becomes

$$
\begin{aligned}
\frac{\mathrm{d} C}{\mathrm{~d} t}(0)= & -\frac{b_{2}}{\operatorname{det} U}(-(\alpha \delta+\beta \sigma))\left(\alpha G F_{0}+\beta G I_{0}\right) \\
& +\frac{(\alpha \delta+\beta \sigma)}{p \operatorname{det} U}\left(-\left(1-\lambda_{1}\right)(2+\gamma) c_{1}\left(\left(a^{2}-b^{2}-1\right) b_{2}-2 a b a_{2}\right)\right. \\
& +b(2+\gamma) c_{1}\left(2 a b b_{2}+a_{2}\left(a^{2}-b^{2}-1\right)\right) \\
& \left.-\left(\left(1-\lambda_{1}\right)^{2}+\lambda_{2}^{2}\right)\left(b_{2}\left(a^{2}-b^{2}-1\right)-2 a b a_{2}\right) c_{1}\right)
\end{aligned}
$$

Now consider the chemo therapy model, when $\nabla>0, \lambda_{+}, \lambda_{-} \neq 0, \alpha \delta+\beta \sigma<0$ and $1, \lambda_{+}, \lambda_{-}$distinct. Define the vector field $X$ by (29). It has flow (30). Here

$$
D^{-1}(A c+c)=\frac{1}{\operatorname{det} D}\left(\begin{array}{c}
0 \\
(2+\gamma) c_{1}(\alpha \delta+\beta \sigma)+\alpha\left(1-\lambda_{-}\right) \delta c_{1}+\beta\left(1-\lambda_{-}\right) \sigma c_{1} \\
-(2+\gamma) c_{1}(\alpha \delta+\beta \sigma)-\alpha\left(1-\lambda_{+}\right) \delta c_{1}-\beta\left(1-\lambda_{+}\right) \sigma c_{1}
\end{array}\right)
$$

The second coordinate here should be equal to

$$
\frac{\left(\lambda_{+}^{2}-1\right) d_{2}}{\ln \lambda_{+}^{2}}
$$

while the third coordinate should be equal to

$$
\frac{\left(\lambda_{-}^{2}-1\right) d_{3}}{\ln \lambda_{-}^{2}}
$$

in order that the time one map of $D X D^{-1}$ is $T^{2}$. Now we can find

$$
\begin{aligned}
\frac{\mathrm{d} C}{\mathrm{~d} t}(0)= & \frac{\mathrm{d}}{\mathrm{d} t}\left(D_{1} \circ \Phi^{X}\right)(0, x) \\
= & \frac{1}{\operatorname{det} D}\left(\left(\alpha G F_{0}+\beta G I_{0}\right)(-(\alpha \delta+\beta \sigma))\left(\ln \lambda_{+}^{2}-\ln \lambda_{-}^{2}\right)\right. \\
& +\left(1-\lambda_{+}\right) \frac{\ln \lambda_{+}^{2}}{\lambda_{+}^{2}-1}\left((2+\gamma) c_{1}(\alpha \delta+\beta \sigma)+(\alpha \delta+\beta \sigma)\left(1-\lambda_{-}\right) c_{1}\right) \\
& -\left(1-\lambda_{-}\right) \frac{\ln \lambda_{-}^{2}}{\lambda_{-}^{2}-1}\left((2+\gamma) c_{1}(\alpha \delta+\beta \sigma)+(\alpha \delta+\beta \sigma)\left(1-\lambda_{+}\right) c_{1}\right)
\end{aligned}
$$

and this is simplified to

$$
\begin{aligned}
\frac{\mathrm{d} C}{\mathrm{~d} t}(0)= & \frac{(\alpha \delta+\beta \sigma)}{\operatorname{det} D}\left(-\left(\alpha G F_{0}+\beta G I_{0}\right)\left(\ln \lambda_{+}^{2}-\ln \lambda_{-}^{2}\right)\right. \\
& \left.-\frac{\ln \left(\lambda_{+}^{2}\right) c_{1}}{1+\lambda_{+}}\left((2+\gamma)+\left(1-\lambda_{-}\right)\right)+\frac{\ln \left(\lambda_{-}^{2}\right) c_{1}}{1+\lambda_{-}}\left((2+\gamma)+\left(1-\lambda_{+}\right)\right)\right)
\end{aligned}
$$

Remark 1 When $\gamma=-3, \alpha=\delta=\sigma=1, \beta=-3.2525$ then $\nabla<0, a=-\frac{1}{2}, b=0.05$, that is $a^{2}-b^{2}>0$. So by the above you can find an affine vector field whose time one map is $T^{2}$. Similarly when $\gamma=-3, \alpha=\delta=\sigma=1, \beta=-\frac{9}{4}$, then $\nabla>0$ and $\lambda_{+}=\frac{1}{2}, \lambda_{-}=-\frac{3}{2}$. So by the above, you have a formula for 
$\frac{\mathrm{d} C}{\mathrm{~d} t}(0)$ on $C=0$.

\section{Escaping Phase Space}

In this section $\mu_{F}=\mu_{I}=0, g=0$. The phase space of our model $T$ of the introduction is $\mathbb{R}_{+}^{3}$. When $\operatorname{det} U \neq 0, \nabla<0, a>0$ integral curves of $B$ from theorem 1 in Larsen (2016), [1], starting in $\mathbb{R}_{+}^{3}$ will always escape phase space for both $t>0$ and $t<0$. Here

$$
F=\left(\begin{array}{ccc}
0 & 0 & 0 \\
0 & a_{1} & b_{1} \\
0 & -b_{1} & a_{1}
\end{array}\right)
$$

and $B=D U \circ F \circ U^{-1}$, where

$$
a_{1}=\frac{1}{2} \ln \left(a^{2}+b^{2}\right), \quad b_{1}=\tan ^{-1}\left(\frac{b}{a}\right) .
$$

$U$ as in section 3. This vector field, $B$, has time one map $T$, see Larsen (2016), [1], or argue as in Section 3.

The purpose of this section is to prove, that there exists a first escape time $t>0$, i.e. the existence of a smallest $t>0$, such that

$$
\Phi^{B}(t, c) \notin \mathbb{R}_{+}^{3}, \quad c=\left(C_{0}, G F_{0}, G I_{0}\right)^{\mathrm{T}} \in \mathbb{R}_{+}^{3}
$$

When $c \in \mathbb{R}_{+}^{3}, \nabla>0, \lambda_{-}, \lambda_{+}>0, \alpha \delta+\beta \sigma<0$, det $D \neq 0$, we prove, that either

$$
\Phi^{B}(t, c) \in \mathbb{R}_{+}^{3}, \quad \forall t>0
$$

or there exists a smallest $t>0$ such that

$$
\Phi^{B}(t, c) \notin \mathbb{R}_{+}^{3}
$$

Proposition 3 Suppose $\operatorname{det} U \neq 0, \nabla<0, a>0$. Given $c=\left(C_{0}, G F_{0}, G I_{0}\right) \in \mathbb{R}_{+}^{3}$ then there exists $t_{1}>0$, $t_{2}<0$ such that

$$
\begin{gathered}
\Phi^{B}\left(t_{i}, c\right)_{1}=0 \\
i=1,2 .
\end{gathered}
$$

Proof. We have the following formula for the flow of $B$

$$
\begin{aligned}
& \Phi^{B}(t, c)_{1}=U_{1} \circ \Phi^{F}\left(t, U^{-1}(c)\right) \\
& =\mathrm{e}^{a_{1} t}\left((1-a)\left(\cos \left(b_{1} t\right) y+\sin \left(b_{1} t\right) z\right)-b\left(-\sin \left(b_{1} t\right) y+\cos \left(b_{1} t\right) z\right)\right)
\end{aligned}
$$

Here

$$
\begin{aligned}
& \left(\begin{array}{l}
x \\
y \\
z
\end{array}\right)=U^{-1}\left(\begin{array}{c}
C_{0} \\
G F_{0} \\
G I_{0}
\end{array}\right), \\
& U_{1}=(0,1-a,-b)
\end{aligned}
$$

and

$$
\Phi^{F}\left(t,\left(\begin{array}{l}
x \\
y \\
z
\end{array}\right)\right)=\left(\begin{array}{c}
x \\
\mathrm{e}^{a_{1} t}\left(\cos \left(b_{1} t\right) y+\sin \left(b_{1} t\right) z\right) \\
\mathrm{e}^{a_{1} t}\left(-\sin \left(b_{1} t\right) y+\cos \left(b_{1} t\right) z\right)
\end{array}\right)
$$


Define

$$
\begin{gathered}
v=(1-a) y-b z=C_{0} \\
w=(1-a) z+b y
\end{gathered}
$$

Since $C_{0}>0$ we can define $\phi \in[0,2 \pi[$ by

$$
\cos \phi=\frac{v}{\sqrt{v^{2}+w^{2}}}, \quad \sin \phi=\frac{w}{\sqrt{v^{2}+w^{2}}}
$$

It follows that we have the following formula

$$
\Phi^{B}(t, x)_{1}=\mathrm{e}^{a_{1} t} \cos \left(b_{1} t-\phi\right) \sqrt{v^{2}+w^{2}}
$$

Since $b_{1}=\tan ^{-1}\left(\frac{b}{a}\right) \neq 0$ the proposition follows.

Remark 2 By the proof we have

$$
t=\frac{\left(p+\frac{1}{2}\right) \pi+\phi}{b_{1}}
$$

implies $\Phi^{B}(t, c)_{1}=0$. Here $p \in \mathbb{Z}$. Let $s_{1}$ denote the smallest positive solution to $\Phi^{B}(t, c)_{1}=0, t>0$.

When $\operatorname{det} D \neq 0, \nabla>0, \lambda_{+}, \lambda_{-}>0, \alpha \delta+\beta \sigma<0$ we have the following proposition using the definitions

$$
\begin{aligned}
& y=C_{0} \frac{1-\lambda_{+}}{\lambda_{-}-\lambda_{+}}-\frac{\alpha G F_{0}+\beta G I_{0}}{\lambda_{-}-\lambda_{+}} \\
& z=-C_{0} \frac{1-\lambda_{-}}{\lambda_{-}-\lambda_{+}}+\frac{\alpha G F_{0}+\beta G I_{0}}{\lambda_{-}-\lambda_{+}}
\end{aligned}
$$

These formulas are explained in the proof of Proposition 4.

Let $B=D \circ F \circ D^{-1}$, where

$$
F=\left(\begin{array}{ccc}
0 & 0 & 0 \\
0 & \ln \lambda_{+} & 0 \\
0 & 0 & \ln \lambda_{-}
\end{array}\right)
$$

$D$ as in section 3. $B$ has time one map $T$, see Larsen (2016), [1], or argue as in section three.

Proposition 4 Suppose det $D \neq 0, \nabla>0, \lambda_{+}, \lambda_{-}>0, \alpha \delta+\beta \sigma<0$. Let $c=\left(C_{0}, G F_{0}, G I_{0}\right) \in \mathbb{R}_{+}^{3}$ be given. (i) If $y<0$, then there exists a unique $t>0$ such that

If $y \geq 0$ then

$$
\Phi^{B}(t, c)_{1}=0
$$

$$
\Phi^{B}(t, c)_{1}>0
$$

for all $t>0$.

(ii) If $z<0$ then there exists a unique $t<0$ such that

$$
\Phi^{B}(t, c)_{1}=0
$$

If $z \geq 0$ then

$$
\Phi^{B}(t, c)_{1}>0
$$


for all $t<0$.

Proof. First of all the flow of $F$ is

$$
\begin{gathered}
\Phi^{F}(t, w)=\left(\begin{array}{c}
\tilde{x} \\
\exp \left(t \ln \lambda_{+}\right) \tilde{y} \\
\exp \left(t \ln \lambda_{-}\right) \tilde{z}
\end{array}\right), \quad w=(\tilde{x}, \tilde{y}, \tilde{z}) \in \mathbb{R}^{3} \\
\left(\begin{array}{c}
\tilde{x} \\
\tilde{y} \\
\tilde{z}
\end{array}\right)=D^{-1}\left(\begin{array}{c}
C_{0} \\
G F_{0} \\
G I_{0}
\end{array}\right)
\end{gathered}
$$

We have the following formula

$$
\begin{aligned}
\Phi^{B}(t, c)_{1} & =D_{1} \circ \Phi^{F}\left(t, D^{-1}(c)\right) \\
& =\exp \left(\left(\ln \lambda_{+}\right) t\right) y+\exp \left(\left(\ln \lambda_{-}\right) t\right) z \\
& =\exp \left(\left(\ln \lambda_{+}\right) t\right)\left(y+\exp \left(\left(\ln \lambda_{-}-\ln \lambda_{+}\right) t\right) z\right)
\end{aligned}
$$

where $D_{1}$ is the first row of $D$. From this equation, (i) follows. For (ii) write

$$
\Phi^{B}(t, c)_{1}=\exp \left(\left(\ln \lambda_{-}\right) t\right)\left(z+\exp \left(\left(\ln \lambda_{+}-\ln \lambda_{-}\right) t\right) y\right)
$$

From this formula, (ii) follows.

Remark 3 In case (i) of the proposition, if $y<0$ we have

$$
t_{1}=\frac{\ln \left(-\frac{y}{z}\right)}{\ln \lambda_{-}-\ln \lambda_{+}}
$$

implies

$$
\Phi_{1}^{B}\left(t_{1}, c\right)=0
$$

In case (ii) of the proposition, if $z<0$ we have

$$
t=\frac{\ln \left(-\frac{z}{y}\right)}{\ln \lambda_{+}-\ln \lambda_{-}}
$$

implies

$$
\Phi_{1}^{B}(t, c)=0
$$

We shall now derive a formula for the first escape time $F E T \in \mathbb{R}_{+}$. To start with, assume that $c \in \mathbb{R}_{+}^{3}$, $\nabla<0, a>0, \operatorname{det} U \neq 0$. Notice that

$$
\begin{aligned}
g_{F}(t) & =\Phi^{B}(t, c)_{2}=(\beta,-\delta, 0) \Phi^{F}\left(t, U^{-1}(c)\right) \\
& =\beta \tilde{x}-\delta\left(\mathrm{e}^{a_{1} t} \cos \left(b_{1} t\right) \tilde{y}+\sin \left(b_{1} t\right) \tilde{z}\right)
\end{aligned}
$$

and

$$
\begin{aligned}
g_{I}(t) & =\Phi^{B}(t, c)_{3}=(-\alpha,-\sigma, 0) \Phi^{F}\left(t, U^{-1}(c)\right) \\
& =-\alpha \tilde{x}-\sigma\left(\mathrm{e}^{a_{1} t}\left(\cos \left(b_{1} t\right) \tilde{y}+\sin \left(b_{1} t\right) \tilde{z}\right)\right)
\end{aligned}
$$


where

$$
\begin{gathered}
\tilde{x}=\frac{1}{\operatorname{det} U}\left(\sigma G F_{0}-\delta G I_{0}\right) b \\
\tilde{y}=-\frac{1}{\operatorname{det} U}\left(\alpha G F_{0}+\beta G I_{0}\right) b \\
\tilde{z}=-\frac{1}{\operatorname{det} U}\left((\alpha \delta+\beta \sigma) C_{0}+(1-a)\left(\alpha G F_{0}+\beta G I_{0}\right)\right)
\end{gathered}
$$

i.e.

$$
\left(\begin{array}{c}
\tilde{x} \\
\tilde{y} \\
\tilde{z}
\end{array}\right)=U^{-1}\left(\begin{array}{c}
C_{0} \\
G F_{0} \\
G I_{0}
\end{array}\right) \in \mathbb{R}^{3}
$$

Compute

$$
\begin{aligned}
& g_{F}^{\prime}(t)=-\delta \mathrm{e}^{a_{1} t}\left(v \cos \left(b_{1} t\right)+w \sin \left(b_{1} t\right)\right) \\
& g_{I}^{\prime}(t)=-\sigma \mathrm{e}^{a_{1} t}\left(v \cos \left(b_{1} t\right)+w \sin \left(b_{1} t\right)\right)
\end{aligned}
$$

where

$$
v=a_{1} \tilde{y}+b_{1} \tilde{z}, \quad w=a_{1} \tilde{z}-b_{1} \tilde{y}
$$

If $(v, w)=(0,0)$ let $s_{F}=+\infty, s_{I}=+\infty$. If $(v, w) \neq(0,0)$ define $\psi \in[0,2 \pi[$ by

$$
(\cos \psi, \sin \psi)=\frac{(v, w)}{\sqrt{v^{2}+w^{2}}}
$$

Then we have the following formulas

$$
\begin{aligned}
& g_{F}^{\prime}(t)=-\delta \mathrm{e}^{a_{1} t} \sqrt{v^{2}+w^{2}} \cos \left(b_{1} t-\psi\right) \\
& g_{I}^{\prime}(t)=-\sigma \mathrm{e}^{a_{1} t} \sqrt{v^{2}+w^{2}} \cos \left(b_{1} t-\psi\right)
\end{aligned}
$$

Assume that $a_{1}<0, \beta \tilde{x} \neq 0$. Then there exists $T>0$ such that

$$
g_{F}(t) \neq 0
$$

for $t \geq T$. If there exists $t_{F} \in[0, T]$ such that

$$
g_{F}\left(t_{F}\right)=0
$$

we claim that there are atmost finitely many such solutions and hence that there exists a smallest $s_{F}>0$ such that

$$
g_{F}\left(s_{F}\right)=0
$$

Assume for contradiction, that there are infinitely many solutions to

$$
g_{F}(s)=0, \quad s \in[0, T]
$$

By (31) there are exactly $n \in \mathbb{N}_{0}$ solutions to

$$
g_{F}^{\prime}(s)=0, \quad s \in[0, T]
$$


Since there are infinitely many solutions to $g_{F}(s)=0, s \in[0, T]$, there exist

$$
t_{1}<t_{2}<\cdots<t_{n+2}
$$

in $[0, T]$ such that

$$
g_{F}\left(t_{i}\right)=0
$$

By the mean value theorem, there exists $\left.\zeta_{i} \in\right] t_{i}, t_{i+1}[$ such that

$$
0=g_{F}\left(t_{i}\right)-g_{F}\left(t_{i+1}\right)=g_{F}^{\prime}\left(\zeta_{i}\right)\left(t_{i+1}-t_{i}\right)
$$

$i=1, \cdots, n+1$. Hence

$$
g_{F}^{\prime}\left(\zeta_{i}\right)=0
$$

$i=1, \cdots, n+1$. A contradiction and there are only finitely many solutions to $g_{F}(s)=0, s \in[0, T]$. If there exists a $t_{F}>0$, such that $g_{F}\left(t_{F}\right)=0$ let $s_{F}>0$ denote the smallest such number, and otherwise let $S_{F}=+\infty$.

If $\beta \tilde{X}=0$ then

$$
g_{F}(t)=-\delta \mathrm{e}^{a_{1} t}\left(\cos \left(b_{1} t\right) \tilde{y}+\sin \left(b_{1} t\right) \tilde{z}\right)
$$

Since $g_{F}(0) \neq 0$, then $\tilde{y} \neq 0$. Define $\tilde{\psi} \in[0,2 \pi[$ by

$$
(\cos \tilde{\psi}, \sin \tilde{\psi})=\frac{(\tilde{y}, \tilde{z})}{\sqrt{\tilde{y}^{2}+\tilde{z}^{2}}}
$$

so

$$
g_{F}(t)=-\delta \mathrm{e}^{a_{1} t} \cos \left(b_{1} t-\tilde{\psi}\right) \sqrt{\tilde{y}^{2}+\tilde{z}^{2}}
$$

By $s_{F}$ denote the smallest positive solution to $g_{F}(t)=0$. Suppose $\beta \tilde{x} \neq 0$ and $a_{1}=0$, if $(\tilde{y}, \tilde{z})=(0,0)$ let $s_{F}=+\infty$, otherwise write (33). If

$$
|\beta \tilde{x}|>\delta \sqrt{\tilde{y}^{2}+\tilde{z}^{2}}
$$

let $s_{F}=+\infty$ otherwise let

$$
b_{1} t_{p}^{ \pm}-\tilde{\psi}= \pm \cos ^{-1}\left(\frac{\beta \tilde{x}}{\delta \sqrt{\tilde{y}^{2}+\tilde{z}^{2}}}\right)+p \pi
$$

$p \in \mathbb{Z}$, so that

$$
g_{F}\left(t_{p}^{ \pm}\right)=0
$$

By $s_{F}$ denote the smallest positive $t_{p}^{ \pm}$. Here

$$
\cos :[0, \pi] \rightarrow[-1,1]
$$

Suppose $\beta \tilde{x} \neq 0, a_{1}>0$. If $(\tilde{y}, \tilde{z})=(0,0)$ let $s_{F}=+\infty$, otherwise write (33). Then there exists $T>0$, such that $g_{F}(T)=0$. By $s_{F}$ denote the smallest positive solution to $g_{F}(t)=0, t \in[0, T]$, arguing as above. If $g_{I}(t)>0$ for all $t>0$ let $s_{I}=+\infty$, otherwise denote by $s_{I}$ the smallest positive solution to $g_{I}(t)=0$. Now define the first escape time FET by

$$
F E T=\min \left\{s_{1}, s_{F}, s_{I}\right\}
$$

We shall now find the first escape time when $\operatorname{det} D \neq 0, \nabla>0, \lambda_{+}, \lambda_{-}>0, \alpha \delta+\beta \sigma<0$. Then we have 


$$
\begin{aligned}
g_{F}(t) & \triangleq \Phi^{B}(t, c)_{2}=D_{2} \circ \Phi^{F}\left(t, D^{-1}(c)\right)=(\beta,-\delta,-\delta) \Phi^{F}\left(t, D^{-1} c\right) \\
& =\beta \tilde{x}-\delta \tilde{y} \exp \left(\left(\ln \lambda_{+}\right) t\right)-\delta \tilde{z} \exp \left(\left(\ln \lambda_{-}\right) t\right)
\end{aligned}
$$

and

where

$$
\begin{aligned}
g_{I}(t) & \triangleq \Phi^{B}(t, c)_{3}=(-\alpha,-\sigma,-\sigma) \Phi^{F}\left(t, D^{-1} c\right) \\
& =-\alpha \tilde{x}-\sigma \tilde{y} \exp \left(\ln \lambda_{+} t\right)-\sigma \tilde{z} \exp \left(\ln \lambda_{-} t\right)
\end{aligned}
$$

$$
\begin{gathered}
\tilde{x}=\frac{1}{\operatorname{det} D}\left(-\sigma\left(\lambda_{+}-\lambda_{-}\right) G F_{0}+\delta\left(\lambda_{+}-\lambda_{-}\right) G I_{0}\right) \\
\tilde{y}=\frac{1}{\operatorname{det} D}\left((\beta \sigma+\alpha \delta) C_{0}+\alpha\left(1-\lambda_{-}\right) G F_{0}+\beta\left(1-\lambda_{-}\right) G I_{0}\right) \\
\tilde{z}=\frac{1}{\operatorname{det} D}\left(-(\beta \sigma+\alpha \delta) C_{0}-\alpha\left(1-\lambda_{+}\right) G F_{0}-\beta\left(1-\lambda_{+}\right) G I_{0}\right)
\end{gathered}
$$

i.e.

$$
\left(\begin{array}{c}
\tilde{x} \\
\tilde{y} \\
\tilde{z}
\end{array}\right)=D^{-1}\left(\begin{array}{c}
C_{0} \\
G F_{0} \\
G I_{0}
\end{array}\right)
$$

Assume in the notation of Proposition 4, that $y<0$ and let

$$
S_{1}=\frac{\ln \left(-\frac{y}{z}\right)}{\ln \lambda \_-\ln \lambda_{+}}
$$

If $y \geq 0$ let $s_{1}=+\infty$. Now compute

$$
\begin{aligned}
g_{F}^{\prime}(t) & =-\delta \tilde{y} \ln \left(\lambda_{+}\right) \exp \left(\left(\ln \lambda_{+}\right) t\right)-\delta \tilde{z} \ln \left(\lambda_{-}\right) \exp \left(\left(\ln \lambda_{-}\right) t\right) \\
& =\exp \left(\left(\ln \lambda_{+}\right) t\right)\left(-\delta \tilde{y} \ln \left(\lambda_{+}\right)-\delta \tilde{z} \ln \left(\lambda_{-}\right) \exp \left(\left(\ln \lambda_{-}-\ln \lambda_{+}\right) t\right)\right)
\end{aligned}
$$

and

$$
g_{I}^{\prime}(t)=-\sigma \tilde{y} \ln \left(\lambda_{+}\right) \exp \left(\left(\ln \lambda_{+}\right) t\right)-\sigma \tilde{z} \ln \left(\lambda_{-}\right) \exp \left(\left(\ln \lambda_{-}\right) t\right)
$$

There are atmost two solutions to $g_{F}(t)=0, t>0$. If there exists $t_{F}>0$ such that $g_{F}\left(t_{F}\right)=0, t_{F}>0$, let $s_{F}>0$ denote the smallest such solution, otherwise let $s_{F}=+\infty$. If there exists $t_{I}>0$ such that $g_{I}\left(t_{I}\right)=0$, $t_{I}>0$, let $s_{I}>0$ denote the smallest such solution, otherwise let $s_{I}=+\infty$. Now define the first escape time, when $\left(s_{1}, s_{F}, s_{I}\right) \neq(+\infty,+\infty,+\infty)$

$$
F E T=\min \left\{s_{1}, s_{F}, s_{I}\right\}
$$

\section{Summary and Discussion}

In this paper we proved that the model of primary and metastatic cancer in Section 2 is bistable, in the sense, that there are exactly two positive singular points. One of them is unstable, and when one of the rate constants is small the other is stable. Then we found formulas for the rate of change of cancer growth for the model $T$ of the introduction, when for $\nabla>0$ the eigenvalues $\lambda_{+}, \lambda_{-}$are nonzero and for $\nabla<0$ when $a^{2}-b^{2}>0$. In section four we proved that there is a first escape time for the flow of the affine vector field associated to $T$ when $\nabla<0$. A similar result when $\nabla>0$ was also treated. 
It would be interesting to figure out what happens if the polynomials $P, P_{M}$ of section 2 are cubic polynomials and not quadratic as in Theorem 1.

\section{About the References}

How do cancer cells coordinate glycolysis and biosynthesis. They do that with the aid of an enzyme called Phosphoglycerate Mutase 1. In the reference [12], the authors suggest a dynamical system for their findings in a figure at the end of the paper. In the reference [13], A. K. Laird showed that solid tumors do not grow exponentially, but rather like a Gompertz function. The publications of the author are concerned with semi Riemannian dynamical systems, e.g. Lorentzian Geodesic Flows, see [14] and electrical network theory of countable graphs, see [15], [16].

\section{References}

[1] Larsen, J.C. (2016) Models of Cancer Growth. Journal of Applied Mathematics and Computing.

[2] Liu, X., Lan, Y., Zhang, D., Wang, K. and Hua, Z.-C. (2014) SPRY1 Promotes the Degradation of uPAR and Inhibits uPAR-Mediated Cell Adhesion and Proliferation. American Journal of Cancer Research, 4, 683-697.

[3] Luo, M. and Fu, L.-W. (2014) Redundant Kinase Activation and Resistance of EGFR-Tyrosine Kinase Inhibitors. American Journal of Cancer Research, 4, 608-628.

[4] John, A. and Bellomo, N. (1997) A Survey of Models for Tumor-Induced Immune System Dynamics. Birkhäuser, Boston.

[5] Geha, R. and Notarangelo, L. (2012) Case Studies in Immunology. Garland Science, Hamden.

[6] Murphy, K. (2012) Immunobiology. 8th Edition, Garland Science, Hamden.

[7] Marks, F., Klingmüller, U. and Müller-Decker, K. (2009) Cellular Signal Processing. Garland Science, Hamden.

[8] Molina-Paris, C. and Lythe, G. (2011) Mathematical Models and Immune Cell Biology. Springer Verlag, New York. http://dx.doi.org/10.1007/978-1-4419-7725-0

[9] Larsen, J.C. The Bistability Theorem in a Cancer Model.

[10] Horn, F. and Jackson, R. (1972) General Mass Action Kinetics. Archive for Rational Mechanics and Analysis, 47, 81116. http://dx.doi.org/10.1007/BF00251225

[11] Sarmah, H.K., Das, M.C. and Baishya, T.K. (2015) HOPF Bifurcation in a Chemical Model. International Journal for Innovative Research in Science and Technology, 1, 23-33.

[12] Hitosugi, T., et al. (2012) Phosphoglycerate Mutase 1 Coordinates Glycolysis and Biosynthesis to Promote Tumor Growth. Cancer Cell, 22, 585-600.

[13] Laird, A.K. (1964) Dynamics of Tumor Growth. British Journal of Cancer, 18, 490-502. http://dx.doi.org/10.1038/bjc.1964.55

[14] Larsen, J.C. (1996) Lorentzian Geodesic Flows. Journal of Differential Geometry, 43, 119-170.

[15] Larsen, J.C. (1991) Dynamical Systems and Semi Riemannian Geometry. Ph.D. Thesis, the Technical University of Denmark, Lyngby.

[16] Larsen, J.C. (1997) Electrical Network Theory of Countable Graphs. IEEE Transactions, Curcuits and Systems: Fundamental Theory and Applications, 44, 1045-1055. 


\section{Submit or recommend next manuscript to SCIRP and we will provide best service for you:}

Accepting pre-submission inquiries through Email, Facebook, Linkedin, Twitter, etc A wide selection of journals (inclusive of 9 subjects, more than 200 journals)

Providing a 24-hour high-quality service

User-friendly online submission system

Fair and swift peer-review system

Efficient typesetting and proofreading procedure

Display of the result of downloads and visits, as well as the number of cited articles

Maximum dissemination of your research work

Submit your manuscript at: http://papersubmission.scirp.org/ 